\title{
Current Situation of Energy Consumption in Guangzhou Railway Station and Analysis on the Potential of Energy Conservation
}

\author{
Kai Fu \& Zhihui Deng \\ School of Mechanical Engineering \\ Southwest Jiaotong University \\ No.111 $2^{\text {nd }}$ Ring Road North Section 1, Chengdu 610031, China \\ E-mail: fk16@163.com
}

\begin{abstract}
According to the questionnaires about the energy consumption situation of Guangzhou railway station, information regarding to the situation of electricity consumption, gas consumption and gas consumption in the station during last two years has been gathered, which serves as the basis for analysis and research, focusing on several main parts of energy consumption in the station like the air-conditioning system and illumination, as well as their components and mutual influence. By gathering and analyzing the data, it has been found that there exist some improper waste phenomena in the designing, daily operation and maintenance of the systems mentioned above in the station. A series of measures for energy saving are brought forward in the paper.
\end{abstract}

Keywords: Railway station, Energy consumption, Air-conditioning system, Energy-conservation

\section{Introduction}

With the rapid development of economy in China, demand for energy is constantly growing. Meanwhile, the conflicts between the shortage of energy and economic growth have become increasingly conspicuous. Buildings, an indispensable part in people's life, with their multiple comprehensive functions, consume energy of a large amount (Jiang, 2005, p.30-31) in modern information and economic society. As large-scale public constructions, railway stations have the common characteristics of other public constructions, for example, automation of equipment, office automation, and communication modernization etc, while it has its own uniqueness as a transportation hub. Public constructions of this kind have their own characteristics like large space, high ratio of windows and walls; high turnover rate of the staff; complex lighting etc. As a result, the consumption of electricity, gas and water is further increased, posing more difficulties in energy conservation. However, Energy-saving design standards for public buildings reported that there still exist huge potential for energy saving. Therefore, the extra-consumption caused by these specialties is a key in the research and analysis on energy-saving of the railway station.

This paper makes an in-depth analysis on the various energy consumption of the Guangzhou railway station. The survey applies the method of direct interview, and collects data through the completion of pre-designed questionnaires which includes basic information of constructions, detailed information about the air-conditioning system, lighting system and power equipment system, the variety of energy usage in the past two years. The basic information includes the building age, areas of major parts, function and use. The details of the air-conditioning system, lighting system and power equipment system include the types of main equipment, model, power, and other technical indicators, as well as the daily management of the maintenance staff. At last, the characters of energy consumption in the station will be analyzed based on these daily operation data. And correspondent plans for energy conservation will be suggested so as to serve as a reference for the energy saving in the station.

\section{Architectural profile and features}

\subsection{Architectural profile}

Guangzhou Railway Station has 4 floors and $36 \mathrm{~m}$ high on the ground and the total building area is $22,360 \mathrm{~m}^{2}$. The first floor on the ground is used for the ticket hall, the waiting room and the entrance hall; the second floor for the waiting room; the third for offices; the fourth for equipment space. The building has inside and outside window shade, ordinary single-layer glass windows of aluminum alloy.

\subsection{Air-conditioning system situation}

The air-conditioned area is $17,020 \mathrm{~m}^{2}$. Air-conditioned region includes waiting room, the hall on the second floor, office 
space, guest rooms, ticketing centers, maternal and child rooms, customs inspection room, which, except the office space, adopt the Fan Coil Unit and Fresh air Unit system, and offices adopt the Split-type air-conditioning. The cold source is the two-centrifugal-water-cooled unit with same specifications. The system uses two pipes and a pump to control water system. Each unit is equipped with a chilled water pump and a back-up one. See the main equipment in Table 1.

\subsection{Ventilation system}

The entrance hall in the railway station uses mechanical ventilation.

\section{Analysis on the energy consumption in Guangzhou railway station}

\subsection{Monthly energy consumption}

See monthly electricity consumption (2006) of Guangzhou railway station in Figure 1.

It can be seen from Figure 1 that the largest electricity consumption was in August; electricity consumption decreased during September to December; from March to July the electricity consumption had no great change; the lowest electricity consumption was in January, and the electricity consumption in February increased greatly compared with that in January. This relates to Guangzhou's climate and the actual situation of the railway station. According to the weather data since the founding of China, the annual average temperature of Guangzhou is $21.9^{\circ} \mathrm{C}$, with July as the month whose average temperature is $28.4^{\circ} \mathrm{C}$, highest in the year. Although temperature is low in January, the average temperature is still above $13^{\circ} \mathrm{C}$. Temperature difference between the lowest and highest in a year is $15^{\circ} \mathrm{C}$. The maximum power consumption in August was due to the fact that the hot weather in summer caused higher indoor heat load than in other months, and a sudden increase of electricity consumption in February was due to the increase of passenger flow in the station before and after the Spring Festival, and the staff changes led to dramatic changes in the indoor loads.

\subsection{Analysis on the structure of building energy consumption}

To get a clear understanding of the electricity consumption of various systems in Guangzhou railway station, the station is divided into four parts: air-conditioning, lighting, power equipment and lifts. The ratio of electricity consumption by four parts is shown in Figure 2.

As can be seen from Figure 2, the energy consumption of air-conditioning system is the largest, accounting for 53\% of the total energy consumption. This is due to the climate characteristics of Guangzhou which decides that the air-conditioning system should run throughout the year, and nearly all interior construction adopts the Fan Coil Unit and Fresh air Unit system. This results in its large total energy consumption. Next is the electricity consumption of power equipment. The station has many lifts and elevators, so it accounts for the relatively large proportion. Lighting takes the minimal share of only $6 \%$, and this is due to the large-scale use of energy-saving lamps and a more reasonable distribution in the station

In order to do further analyze energy consumption of air conditioning, Figure 3 shows the energy consumption ratio of various parts in air-conditioning system.

It is shown in Figure 3 that centrifugal water-cooled unit shares the largest part, reaching $45 \%$. The proportion of pumps and mechanical ventilation is more or less the same, $21 \%$ and $19 \%$ respectively. The ratio of FCU in air-conditioning system is the least.

\section{Analysis on the potential of energy saving}

It can be found through the above analysis that the air-conditioning, lighting, power equipment, and elevators are four major parts of the electricity consumption. Based on the running record of the year and other relative data analysis about the air-conditioning system, lighting system and office equipment, there exists irrationality and waste of energy in design, operation and management of the air-conditioning system, power equipment system, and then there is great potential for energy conservation. Improving the running and management of lighting, office equipment .etc is also an important energy-saving method.

\subsection{Energy-saving of the air-conditioning system}

Air-conditioning system is the largest part of energy consumption. According to the analysis on the parts of air-conditioning system, we can see that hot and cold source is the largest part of power consumption of the air-conditioning system. Therefore, choosing cold and hot source unit with high COP value plays a key role in improving energy-efficiency of the air-conditioning system. Secondly, air-conditioning system also needs to improve everyday operation and maintenance. The pumps are more consumptive of energy, so we can switch to the combined operation of a variable frequency pump and a general water pump. This method is secure, stable and easy for management, and the running of water pump can be changed in accordance with the changes of the construction load so as to improve the operation of air-conditioning system to save energy. 


\subsection{Energy-saving in power equipment system}

The power equipment system is the second largest part in electricity consumption, so choosing energy-saving equipment and improving the system's management and maintenance are major ways for reducing the building energy consumption.

\subsection{Energy-saving in lighting system}

Generally speaking, the lighting load in railway station is rather large, and this is a very important aspect on building energy-efficiency. Take the waiting room in Guangzhou railway station as an example. The area of the waiting room is $7020 \mathrm{~m}^{2}$. There are about 1297 energy-saving lamps and 818 fluorescent tubes in the room. The lighting load per unit area is about $4.1 \mathrm{~W} / \mathrm{m}^{2}$, slightly higher than the railway passenger station building design codes. Though the building uses a large number of energy-saving lamps, it's not enough, and the design and control system is unreasonable. Therefore, we should choose a new type of energy-saving light source instead of fluorescent tubes, use intelligent lighting control system, improve the management and utilization of the system, optimize the quality of electricity, and save lighting electricity to achieve the best lighting effect. In addition, to enhance and train the staff's awareness of energy-saving is an important measure to reduce the power consumption (Zhang, 2006).

\section{Conclusion}

Through analyzing the whole-year situation of building energy consumption in Guangzhou railway station, we can find that: (1) the air-conditioning, lighting, power equipment, elevators constitute the four major parts of the electricity consumption. (2) Energy consumption of the lighting system in Guangzhou railway station is relatively small, but there is potential for further improvement, so more attention should be paid to it. (3) Energy consumption of the air-conditioning in Guangzhou railway station is the largest, and the energy consumption of the hot and cold source unit accounts for $45 \%$ in the energy consumption of air conditioning. This is a key point in energy saving. (4) There exist irrationality and waste phenomena in the design, operation and management of air-conditioning, lighting and power equipment. It proves that there is great potential for energy conservation.

\section{References}

Yi Jiang. (2005). Current building energy consumption in China and effective energy efficiency measures. $H V \& A C$, 2005, 35(5): 30-31.

Construction Ministry of PRC. (2005). Energy-saving design standards for public buildings. China Building Industry Press.

Railways Ministry of PRC, Construction Ministry of PRC. (1996). Railway passenger station building design codes.

Liying Zhang. (2006). Energy-saving on Railway Lighting System. Ningxia Machinery, 2006, 01.

Table 1. Main air-conditioning equipment in Guangzhou railway station

\begin{tabular}{|l|l|l|}
\hline Equipment Name & Power & Number \\
\hline Centrifugal Water-cooled Unit & Cooling capacity $3059 \mathrm{kw}$,electric power $530 \mathrm{kw}$ & 2 \\
\hline Freezing Water Pump & Rated Power $90 \mathrm{kw}$ & 3 \\
\hline Cooling Water Pump & Rated Power $90 \mathrm{kw}$ & 3 \\
\hline Fan Coil Unit & Total Power $494.825 \mathrm{kw}$ & 124 \\
\hline Tank Lifting Air-conditioner Unit & Rated Power $3 \mathrm{kw}$ & 1 \\
\hline
\end{tabular}




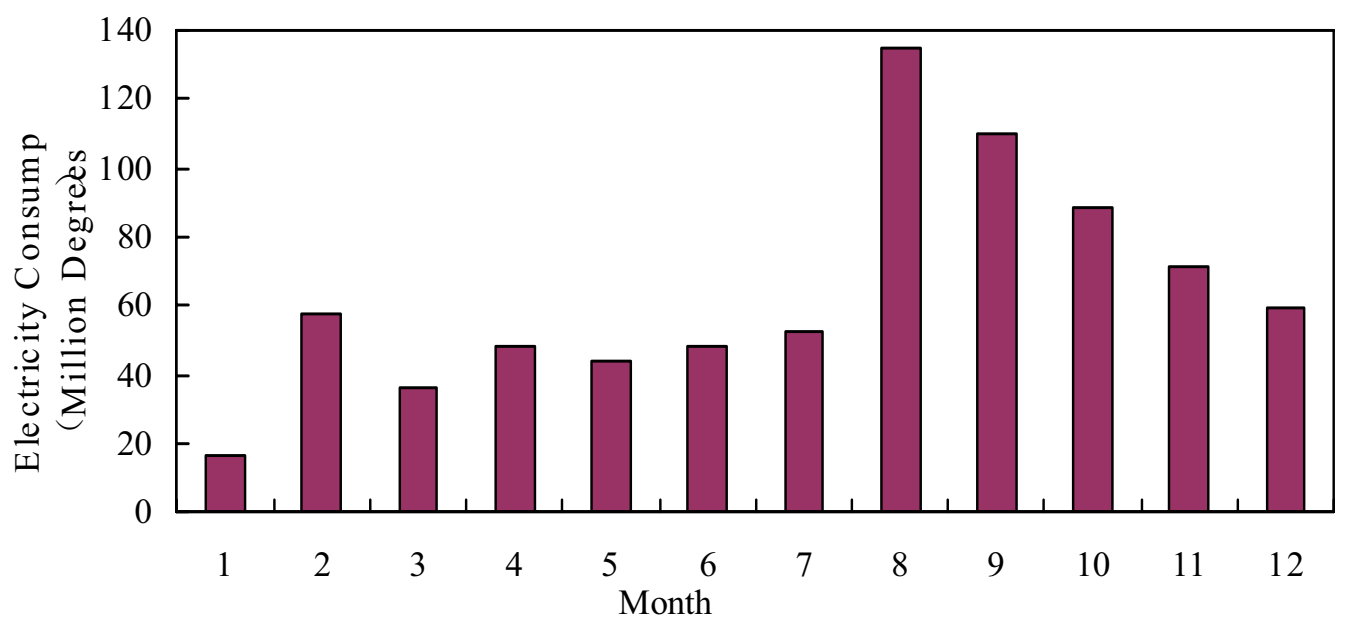

Figure 1. Monthly Electricity Consumption

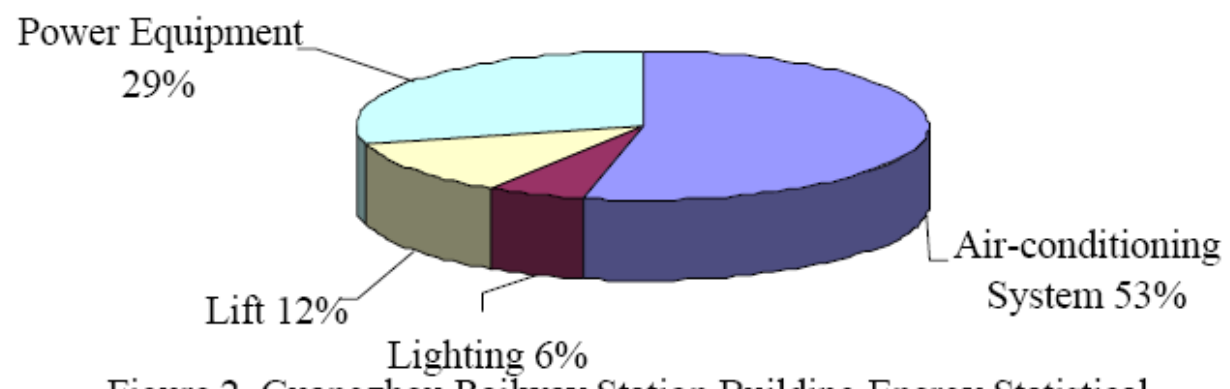

Figure 2. Guangzhou Railway Station Building Energy Statistical

Classification

Mechanical

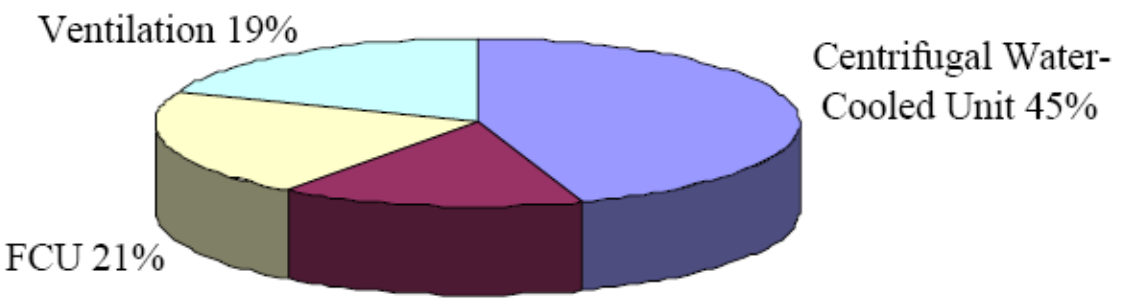

Freezing and

Cooling Pump15\%

Figure 3. The Energy Consumption Ratio of the Various Parts of Airconditioning System 